\title{
Linx
}

Revue des linguistes de l'université Paris X Nanterre

$74 \mid 2017$

Claudine Normand, une vie dans le langage

\section{Greimas lecteur de Saussure}

Michel Arrivé

\section{OpenEdition}

Journals

Édition électronique

URL : http://journals.openedition.org/linx/1762

DOI : 10.4000/linx.1762

ISSN : 2118-9692

\section{Éditeur}

Presses universitaires de Paris Nanterre

\section{Édition imprimée}

Date de publication : 31 juillet 2017

Pagination : 163-173

ISSN : 0246-8743

Référence électronique

Michel Arrivé, «Greimas lecteur de Saussure », Linx [En ligne], 74 | 2017, mis en ligne le 15 mars 2018, consulté le 01 mai 2019. URL : http://journals.openedition.org/linx/1762 ; DOI : 10.4000/linx.1762

Ce document a été généré automatiquement le 1 mai 2019.

Département de Sciences du langage, Université Paris Ouest 


\title{
Greimas lecteur de Saussure
}

\author{
Michel Arrivé
}

\section{Introduction}

1 J'interviens ici en historien de la linguistique et de la sémiotique. C'est en cette qualité que je décrirai les positions prises par Greimas à propos de Saussure. Il est possible qu'on trouve dans mon exposé quelques critiques à l'égard de ces positions. Elles ne sont formulées que par rapport au texte de Saussure, et ne visent en rien la place qu'elles prennent dans l'appareil théorique construit par Greimas, qui a le droit, comme tout un chacun, d'utiliser à sa façon les théories antérieures aux siennes.

2 Greimas avait deux bonnes raisons de porter à l'œuvre de Saussure un intérêt déterminé. C'était d'abord sa qualité de linguiste. Greimas a en effet toujours revendiqué cette qualité. Je cite les propos qu'il a tenus, en 1983, lors du colloque qui, à Cerisy-la-Salle, lui était consacré :

Même si maintenant les linguistes me rejettent et ne me considèrent pas comme l'un des leurs, moi, je prétends être linguiste dans mes origines et dans la façon de conduire ma pensée. Je crois que j'ai toujours cherché à prendre en compte la totalité de l'histoire de la linguistique, essentiellement en tant que philosophie du langage, passage de cette philosophie vers une science du langage. (Greimas, in Arrivé et Coquet, 1983-1987 : 305-306).

Deuxième raison pour Greimas de s'intéresser à Saussure : sa qualité de sémioticien. C'est ici le Saussure initiateur de la sémiologie qui est convoqué. En 1956, Greimas écrit :

De la confrontation des résultats d'ordre méthodologique obtenus par l'école de Focillon et des intuitions nombreuses contenues dans l'œuvre de Malraux avec les principales acquisitions de la linguistique structurale, de l'extension du saussurisme à la musicologie, où la conception de la musique en tant que langage paraît aller de soi, sortirait certainement, en même temps qu'une meilleure compréhension de problèmes propres à chaque domaine, une sémiologie générale pressentie et souhaitée par F. de Saussure. (Greimas, 1956-2000 : 377)

Greimas prend des précautions à l'égard de la sémiologie saussurienne : elle n'est, selon lui, que " pressentie et souhaitée ». Les deux adjectifs sont peut-être un peu insuffisants, 
même si, en 1956, on ne pouvait tenir compte que du $C L G^{1}$ : la sémiologie s'y trouve définie et programmée, plutôt que " pressentie et souhaitée ». Mais ce n'est pas là le plus important. Ce qui compte, c'est que la disjonction entre le Saussure linguiste et le Saussure sémiologue est tenue par Greimas comme artificielle. Rien d'étonnant dans le refus de cette distinction : dans sa Sémantique structurale, Greimas « suspend la distinction entre la sémantique linguistique et la sémiologie saussurienne »(Greimas, 1966: 8-9). Saussure lui aussi envisage comme possible cette "suspension " : c'est l'une des rencontres solides entre les deux appareils théoriques. Mais l'une des seules. Car pour le reste Greimas s'éloignera progressivement d'un saussurisme auquel il n'a d'ailleurs pas adhéré d'emblée.

Pour décrire l'itinéraire saussurien de Greimas, il est indispensable d'envisager les faits du point de vue historique. Je vais donc distinguer quatre étapes successives: 1 . la préhistoire; 2. l'époque des thèses de Greimas; 3. l'article de 1956 sur Saussure; 4. l'époque de la sémiotique.

\section{La préhistoire: Greimas très jeune linguiste en France}

6 Greimas évoquera lui-même, en 1956, les premiers temps de son statut de linguiste :

Reflétant la conviction à peu près unanime de ses maîtres, un jeune linguiste de 1935 avait encore tendance à considérer avec dédain les travaux des écoles de Genève et de Prague, dont l'ésotérisme, disait-on, cachait mal les spéculations purement théoriques, contraires aux faits linguistiques positifs et au bon sens le plus élémentaire (Greimas 1956-2000 : 371)

7 Greimas évoque de façon rapide, mais exacte ce qu'était, dans l'immédiat avant-guerre, l'attitude des linguistes qui étaient ses maîtres au moment du début de ses études en France. L'excellent Antonin Duraffour, avec qui il travailla à Grenoble, de 1936 à 1939, sur des problèmes de préhistoire du lexique, était un linguiste de terrain, spécialisé dans la description, surtout phonétique, des parlers franco-provençaux. C'est lui qui est qualifié de «maître remarquable » dans le texte de 1983-1987, p. 302. Ses travaux sont effectivement au plus haut point intéressants, mais ne comportent, si j'ai bien lu, aucune allusion à Saussure, bien qu'ils datent du début des années 1930, époque où le Cours était déjà bien connu en France par plusieurs linguistes. Ce n'était pas encore le cas pour le très jeune linguiste qu'était alors Greimas - il n'avait que dix-huit ans! Il ne faisait que suivre l'exemple de ses maîtres français, linguistes de terrain peu portés sur les problèmes théoriques.

\section{L'époque des deux thèses de Greimas}

8 Avançons de douze ans dans les travaux de Greimas. L'attitude qu'il manifeste à l'égard de Saussure dans ses deux thèses soutenues à la Sorbonne en 1948 est étonnante. Elles décrivent le vocabulaire de La mode en 1830. Elles ont été publiées sous ce titre en 2000. La bibliographie, très abondante, ne cite pas le CLG, qui n'est jamais allégué dans aucun des deux ouvrages. L'attitude adoptée dans les deux textes à l'égard des faits de lexique étudiés est double et, d'une certaine façon, contradictoire. En effet, Greimas adopte parfois des positions saussuriennes : il revendique pour son étude un aspect «statique ", 
en «évitant autant que possible le point de vue historique » (2000: 7). Attitude constamment maintenue, à quelques entorses près, le plus souvent expliquées et justifiées. La distinction semble bien être celle que Saussure introduisait, quarante ans avant, en opposant les points de vue synchronique et diachronique. Mais Greimas ne retient pas la terminologie saussurienne, et s'en tient à l'opposition statique/historique, comme s'il souhaitait effacer toute référence explicite au CLG. C'est sans doute marque de prudence à l'égard du directeur de la "thèse principale ", Charles Bruneau. Ce fidèle élève et successeur de Ferdinand Brunot, n'avait pas pour Saussure l'antipathie déterminée de son prédécesseur. Mais il avait peu d'intérêt pour lui. Quant au directeur de la " thèse complémentaire ", c'était Robert-Léon Wagner, qui cite et commente longuement Saussure dans ses publications de cette époque et ne pouvait manquer d'en recommander la lecture à ses doctorants. Quoi qu'il en soit, le Greimas de cette époque s'oppose presque littéralement, sur d'autres problèmes, à Saussure. Ainsi il prend le parti de se «tenir le plus près possible des choses : prendre pour point de départ le monde des réalités et non celui des mots " (Greimas 2000:7). On est aux antipodes absolus tant des positions de Saussure que de celles que Greimas prendra dès la Sémantique structurale. Je rappelle ces positions :

La reconnaissance de la clôture de l'univers sémantique implique, à son tour, le rejet des conceptions linguistiques qui définissent la signification comme la relation entre les signes et les choses, et notamment le refus d'accepter la dimension supplémentaire du référent, qu'introduisent, en matière de compromis, les sémanticiens "réalistes " (Ullmann) dans la théorie saussurienne du signe, ellemême sujette à caution : elle ne représente qu'une des interprétations possibles $d u$ structuralisme de Saussure. Car se référer aux choses pour l'explication des signes ne veut rien dire de plus que tenter une transposition, impraticable, des significations contenues dans les langues naturelles en ensembles signifiants non linguistiques: entreprise, on le voit, de caractère onirique. (Greimas 1966 : 13-14).

À cette époque Greimas est devenu non seulement saussurien, mais post-saussurien, puisqu'il met en cause comme « elle-même sujette à caution la théorie saussurienne du signe ». Je reviendrai sur ce point plus tard.

Mais je m'en tiens pour l'instant au texte des deux thèses. On a l'impression que le Greimas de cette époque connait, par la rumeur, certains aspects de l'enseignement de Saussure, mais l'a lu de façon superficielle, au point de ne pas apercevoir l'un des aspects principaux de sa réflexion, l'exclusion de l'« objet désigné », qui n'a pas encore pris le nom de référent. Quoi qu'il en soit, il le considère encore de façon légèrement méfiante, et croit inutile, ou, peut-être, imprudent de le citer dans une thèse, même quand il se situe sur des positions théoriques voisines.

\section{L'article de 1956 sur Saussure}

11 Huit ans plus tard, les choses ont changé du tout au tout. Greimas publie dans Le Français moderne, en juillet 1956, un article intitulé «L'actualité du saussurisme. À l'occasion du $40^{\mathrm{e}}$ anniversaire de la publication du Cours de linguistique générale ». Greimas se révèle dans ce texte très bon connaisseur du texte saussurien et de l'influence qu'il a commencé à exercer sur les sciences humaines de l'époque. Que s'est-il passé entre 1948 et 1956 ? Greimas, toujours assez discret sur son autobiographie intellectuelle, n'en dit pas grandchose. On peut se laisser aller à deux hypothèses, propres d'ailleurs à se cumuler. 
Avec Georges Matoré, Greimas a commencé à travailler sans doute un peu avant l'achèvement de ses thèses, dans l'immédiat après-guerre. Il a écrit avec lui un article, publié dans les Romanische Forschungen, dès 1950 : «La méthode en lexicologie ». C'était le premier moment d'un projet plus vaste, celui de fonder la lexicologie. Greimas rappelle cet épisode en 1983-1987. Il le présente, au même titre que ses thèses de 1948, comme un échec, mais un échec d'une certaine façon positif, puisqu'il l'a détourné des « vieilleries » du champ notionnel et du champ lexical :

Pour moi, la non-pertinence du niveau des signes, je l'ai vécue dans mon expérience lexicologique, parce que c'est la lexicologie que nous avons cherché à fonder avec Georges Matoré dans les années 1940-1950. Un tel constat, vous le comprenez, met tout de suite dans un climat de refus de toute notion de champ notionnel, de champ lexical, etc., ces vieilleries qui traînent encore autour de nous. (1983-1987 : 303 ; un peu plus loin dans le même texte, p. 325, il dira, avec la violence inchangée du souvenir de l'échec, que « la lexicologie ne peut pas être validée, qu'il faut la jeter à la poubelle de l'histoire pour passer à la sémantique »)

Remarquons au passage le coup de patte donné au "niveau des signes » : il vise Saussure, déjà atteint, on vient de le voir, par une mise en cause un peu plus explicite dans Sémantique structurale. Mais c'est surtout la lexicologie à la Matoré qui se trouve atteinte : le livre prévu paraîtra, en 1953, sous la signature du seul Matoré. Greimas n'y est cité, dans l' " Avant-Propos », que comme « lecteur du manuscrit ».

Échec, donc, mais positif, et même doublement positif. Car en même temps qu'il a fait connaître à Greimas l'épreuve de la tentative avortée, Matoré lui a fait lire le Cours de linguistique générale, qui est l'une des références principales de La méthode en lexicologie : un chapitre entier de l'ouvrage dont Greimas, on vient de le voir, a lu le manuscrit est consacré au CLG, pour l'essentiel à l'opposition synchronie/diachronie, qui se trouve à la fois posée comme fondamentale et critiquée par les excès auxquels elle pourrait donner lieu dans le domaine de la lexicologie.

C'est donc là l'une des origines, sans doute la première, de l'attention portée à Saussure par Greimas. La seconde vient de la rencontre de Greimas avec Barthes, en 1949, à l'Université d'Alexandrie, où ils viennent l'un et l'autre d'être nommés. Cette fois Greimas est un peu plus explicite, au point d'escamoter complètement Matoré. Et, en dépit de son aversion avouée pour la chronologie, il établit la succession des lectures que les deux jeunes professeurs font en commun :

Bien plus important, la découverte de Saussure que nous avons faite en commun avec Barthes - Saussure, puis Jakobson, Lévi-Strauss, et Hjelmslev ensuite. En tout cas il est clair pour moi que j'ai connu Hjelmslev avant l'histoire du canal de Suez (1956), date de repérage, parce qu'à ce moment je me trouvais en Égypte. (1983-1987 : 304)

Quoi qu'il en soit des origines de sa lecture de Saussure, Greimas semble bien en 1956 être devenu pleinement saussurien. Il se plaint même du peu d'influence que Saussure exerce en France sur l'évolution de la linguistique. Il exagère un peu. De nombreux grammairiens font intervenir les concepts saussuriens dans leurs analyses: je ne cite pour mémoire que Guillaume, Damourette et Pichon, Tesnière, Gougenheim et Wagner, tous avant 1939 (voir Arrivé 2012). Sans parler de Martinet, en linguistique générale. Mais Greimas tenait Martinet en piètre estime. 
Dans son article, Greimas décrit avec clarté et alacrité les pièces essentielles de l'appareil théorique mis en place dans le CLG. Il analyse d'autre part avec une acuité très remarquable l'influence du Cours sur plusieurs secteurs des sciences humaines de l'époque. C'est même l'enjeu essentiel de l'article tel que Greimas le programme :

Les lignes qui suivent, loin d'esquisser une nouvelle apologie, voudraient plutôt montrer l'efficacité de la pensée de F. de Saussure qui, dépassant les cadres de la linguistique, se trouve actuellement reprise et utilisée par l'épistémologie générale des sciences de l'homme. (1956-2000: 372)

19 Conformément à ce programme, Greimas envisage avec optimisme l'extension des points de vue saussuriens à l'ensemble des sciences humaines. Il énumère la philosophie de Maurice Merleau-Ponty, l'ethnologie de Claude Lévi-Strauss, l'histoire de l'art et l'histoire tout court, la musicologie. Il évoque aussi la littérature. Il envisage une « sémiologie générale ». Une seule lacune : la psychanalyse, notamment Lacan, que Greimas n'aime pas.

\section{L'époque de la sémiotique}

Quel usage Greimas fait-il lui-même de Saussure dans l'élaboration, quelques années plus tard, de la sémantique structurale, puis de la sémiotique ? Il est beaucoup plus réservé, et la portée des réserves qu'il formule est de plus en plus étendue.

21 Dès les années de rédaction de Sémantique structurale - sans doute à partir de 1963, certainement en 1964 - Greimas prend ses distances avec Saussure. Les réserves formulées sur la théorie saussurienne du signe se précisent et s'explicitent. C'est en réalité non seulement le concept de signe qui est mis en cause, mais aussi celui, fondamental chez Saussure, de « système de signes »:

La langue n'est pas un système de signes, mais un assemblage - dont l'économie reste à préciser - de structures de signification. (1966:20)

22 Sans que le nom de Saussure soit ici allégué, Greimas vise ici l'illustre passage du CLG qui définit la langue comme l'un des «systèmes de signes » objets futurs de la sémiologie. Il met en cause le principe de l'arbitraire du signe, et évoque avec précaution le problème, évacué par Saussure, de l'origine du langage :

Il serait hors de propos de soulever ici le problème des origines du langage. Notons, cependant, que la reconnaissance des variations concomitantes des modèles phonologique et sémiologique apporte des éléments nouveaux au dossier, considéré jusqu'à présent comme inactuel. (1966:63).

Les « variations concomitantes des modèles phonologiques et sémiologiques ", c'est une autre formulation de la " motivation du signe ». Le mode d'approche du problème des origines qui se trouve ainsi envisagé semble bien être, retraduit dans la théorie du " proto-sémantisme » de Pierre Guiraud (notamment dans 1967-1986), le bon vieux modèle onomatopéique, modifié par la substitution du " modèle sémiologique » au référent des anciennes théories. Dans le Dictionnaire raisonné de la théorie du langage, on aperçoit l'ébauche d'une contradiction entre l'article arbitraire, dans l'ensemble conforme, en dépit de certaines réserves de détail, à l'enseignement du CLG et l'article motivation, où les auteurs, Greimas et Courtès, reviennent sur « l'approche de P. Guiraud, qui, par-delà les imitations servant à produire des morphèmes isolés (onomatopées), met en évidence l'existence de structures morphophonologiques (du type " tic "/ " tac"), susceptibles de produire des familles entières de mots et de les articuler, en même temps, au niveau 
sémantique, compte tenu des oppositions phonologiques $(/ \mathrm{i} / \mathrm{vs} / \mathrm{a} /)$ » (Greimas et Courtès $1979:$ :240).

C'est là une mise en cause explicite du principe de l'arbitraire du signe. Elle ne s'accompagne pas de ce qui serait, de façon totalement cohérente, une de ses implications : la mise en cause du concept de valeur. Comment le penser sans poser l'arbitraire du signe? C'est, pour Saussure, une impossibilité. Cependant, le concept de valeur donne lieu à une entrée très saussurienne dans le Dictionnaire.

Qu'en est-il maintenant du Saussure fondateur de la sémiologie, à supposer qu'il soit possible de le distinguer du Saussure linguiste ? C'est ici le Greimas de 1983, qui prend la parole, de façon plutôt négative :

Par exemple ce que Saussure dit à propos de la sémiologie, c'est intéressant évidemment, mais c'est anecdotique ; ça fait deux phrases, on ne peut pas faire de la sémiologie avec ça, pas plus que de la sémiotique d'ailleurs. (1983-1987 : 306).

S'ensuit, pour la première fois, à ma connaissance, dans les écrits de Greimas la disjonction entre le Saussure du Mémoire de 1878 et celui du CLG des années 1907-1911 et de son édition de 1916 :

Ce qui est capital dans l'œuvre de Saussure, c'est son Mémoire, et la façon dont il a résumé tout le XIX ${ }^{\text {ème }}$ siècle dans le comparatisme linguistique : c'est son idée de traiter un système comme un ensemble de corrélations. C'était déjà de la sémiotique. Le grand Saussure, il est là ! Ensuite, on peut s'amuser avec signifiant/ signifié, mais à ce jeu, on crée la possibilité de bien des déviations, Saussure luimême a commencé avec 'arbre' comme image psychologique, 'arbre' comme concept, c'est de la plaisanterie - avec cela on ne peut pas faire de la sémiotique. ( ibid.)

Propos assez complexes, où se décèlent des imprécisions sur le statut historique du Mémoire et ce qui est peut-être une erreur, au moins par omission, sur la fonction conférée au «concept» de l'« arbre » dans la définition du signe. J'ajoute que je reste surpris de constater que Greimas semble n'avoir jamais eu aucune curiosité pour la recherche sémiologique de Saussure sur la légende. Elle était pourtant révélée, par Godel dès 1957, quoique de façon très discrète, puis par Starobinski à partir de 1964 et surtout en 1971, en même temps que la recherche sur les Anagrammes, dont Greimas ne souffle mot non plus.

Pour conclure, le mieux est sans doute de citer le Greimas de 1985. À propos du très suggestif « Retour à Saussure? » de Claude Zilberberg, il fait allusion au geste commun d'Oswald Ducrot et de Claude Zilberberg:

Les deux linguistes balaient d'une main le Cours, usurpateur d'une réputation ambiguë, pour installer à sa place le Système. (1985:3).

S'ensuit immédiatement un point de vue complémentaire, déjà aperçu dans certains des textes précédemment cités. Il est, certes, présenté comme celui de Zilberberg. Mais il serait sans doute facile de montrer qu'il est aussi celui qu'adopte Greimas :

Une relecture de Saussure n'est possible qu'à travers Hjelmslev, seul héritier légitime, un Hjelmslev qui ne se trouve pas tout à fait à l'endroit où nous l'avons situé. Un glossaire hjelmslevien pour lire les intuitions fondamentales du jeune Saussure. (ibid.)

30 Telle qu'elle s'exprime à ce moment, la position de Greimas consiste donc à éliminer le Cours et à lui substituer le Système. Mais le Système lui-même n'est à son sens « lisible » que par l'entremise du « glossaire » hjelmslevien. 


\section{Conclusion}

31 On voit l'étendue des problèmes auxquels nous a amené l'exercice au départ très modeste que j'ai entrepris de faire. Il nous conduit en effet à deux tâches difficiles, mais nécessaires. La première serait de réfléchir sur la pertinence de ce rééquilibrage de l'œuvre de Saussure auquel Greimas s'est livré au cours de sa réflexion. La seconde serait de repérer, cette fois dans le texte de Greimas, les effets exercés par ce Saussure rééquilibré et hjelmslevisé.

\section{BIBLIOGRAPHIE}

Arrivé, M., 2007, À la recherche de Ferdinand de Saussure, Paris, PUF.

Arrivé, M., 2012, « Saussure dans les grammaires françaises de l'entre-deux-guerres », in B. Colombat, J.-M. Fournier, et V. Raby, Vers une histoire générale de la grammaire française, Paris, Honoré Champion, p. 169-189.

Arrivé, M. et Coquet, J.-C., 1983-1987, Sémiotique en jeu. À partir et autour de l'œuvre d'A.J. Greimas, Paris/Amsterdam/Philadelphie, Hadès-Benjamins.

Godel, R., 1957, Les sources manuscrites du Cours de linguistique générale de Ferdinand de Saussure, Genève, Droz.

Greimas, A.-J., 1948, Thèses de doctorat d'état, voir Greimas 2000.

Greimas, A.-J., 1956-2000, « L'actualité du saussurisme (à l'occasion du 40 ème anniversaire de la publication du Cours de linguistique générale) », Le Français moderne, XXIV, $\mathrm{n}^{\circ} 3$, juillet, $\mathrm{p}$. 191-203. In Greimas 2000 : 371-382.

Greimas, A.-J., 1966-1986, Sémantique structurale, Paris, Larousse, puis PUF.

Greimas, A.-J., 1983-1987, « Postulats, méthodes et enjeux : Greimas mis à la question », in M. Arrivé, et J.-C. Coquet, 1983-1987, p. 299-330.

Greimas, A.-J., 1985, « Avant-propos » à Zilberberg 1985 : 3-4.

Greimas, A.-J., 2000, La mode en 1830, Paris, PUF (publication des deux thèses de Greimas, voir la note 1$)$.

Greimas, A.-J.et CourtÈs, J., 1979, Sémiotique. Dictionnaire raisonné de la théorie du langage, Paris, Hachette.

Guiraud, P., 1967-1986, Structures étymologiques du lexique français, Paris, Payot.

Matoré, G., 1948-1953, « Remarques sur des thèses récentes de lexicologie du français moderne [celles de Greimas] », dans G. Matoré 1953, p. 118-123.

Matoré, G., 1953, La méthode en lexicologie. Domaine français, Paris, Marcel Didier.

Saussure, F. de, 1878-1922-1984, Mémoire sur le système primitif des voyelles dans les langues indoeuropéennes, in Recueil des publications scientifiques, Genève-Paris, Slatkine reprints, p. 1-268. 
Saussure, F. de, 1916-1922-1985, Cours de linguistique générale, Lausanne et Paris, puis Paris, Payot. Starobinski, J., 1971, Les mots sous les mots. Les anagrammes de Ferdinand de Saussure, Paris, Gallimard.

Tesnière, L., 1959, Éléments de syntaxe structurale, Paris, Klincksieck.

Zilberberg, C., 1985, « Retour à Saussure? », Actes sémiotiques, VII, 63.

\section{NOTES}

1. Ce n'est en effet qu'en 1957 que Godel signale, dans ses Sources manuscrites du Cours de linguistique générale, l'existence des travaux sémiologiques de Saussure sur la légende germanique.

\section{RÉSUMÉS}

Les positions prises par Greimas à l'égard de Saussure (c'est-à-dire du CLG dans sa version standard, seul texte auquel il se réfère) évoluent avec le temps. On distingue les moments suivants : (1)L'époque des deux thèses de Greimas (1948, sur le vocabulaire de la mode en 1830) ne comporte aucune référence explicite à Saussure, même si certains points de méthode évoquent le CLG ; (2)L'article de 1956 sur «L'actualité du saussurisme » est au contraire l'un des moments les plus importants de l'analyse de l'influence du CLG sur différents plans de la réflexion en sciences humaines et sur le développement du structuralisme ; (3) La suite de la réflexion de Greimas se caractérise par une prise en compte raisonnée et critique de la réflexion de Saussure, notamment autour du concept de signe.

INDEX

Mots-clés : sémiologie, ethnologie, philosophie, signe, structuralisme.

\section{AUTEUR}

\section{MICHEL ARRIVÉ}

Université Paris Nanterre, Modyco 